\title{
Simulation und Analyse komplexer Energiesysteme
}

\section{Sehr geehrte, liebe Leserinnen und Leser!}

\author{
L. Fickert OVE
}

Online publiziert am 6. Dezember 2014

(c) Springer Verlag Wien 2014

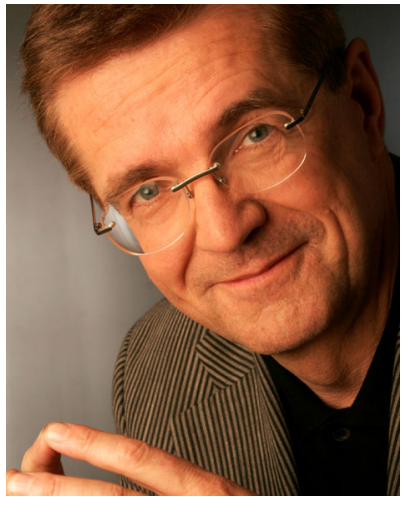

Univ.-Prof. Dr. Lothar Fickert
Die großen Beiträge der Elektrotechnik zu unserer Zivilisation liegen auf den Gebieten der Informationsund Kommunikationstechnik sowie auf der zunehmenden Verbreitung der elektrischen Energie als energetischer Grundversorgung, wobei festzustellen ist, dass die elektrische Energieversorgung für die IKT-Technologien eine essenzielle Voraussetzung darstellt.

Bei der Technik der elektrischen Energienetze ist heute wieder der Simulation und Analyse komplexer elektrischer Energiesysteme zunehmend Aufmerksamkeit zu widmen, da bekanntermaßen die Erzeugung und der Verbrauch elektrischer Energie räumlich disloziert sind und - bei dargebotsabhängiger Einspeisung - auch räumlich-zeitliche Verschiebungen auftreten. In diesem Sinne beschreibt das vorliegende Heft aktuelle Forschungsthemen auf dem Gebiet der elektrischen Energiesysteme. Die Beiträge dieses Heftes lassen sich den Themenkreisen

- Lastfluss

- Kurzschluss und Erdschluss

- Netzdynamik

- Beeinflussungen

zuordnen.

Ausgehend von der Erkenntnis, dass die Hauptaufgabe der elektrischen Energienetze in der zeit- und qualitätsgerechten Versorgung der Verbraucher mit elektrischer Energie besteht, widmet sich der erste Artikel den Gleichzeitigkeitsfaktoren in der elektrischen Energieversorgung, wobei konventionelle Ansätze der Belastungsanalyse mit einem probabilistischen Ansatz aufgrund von realen Datensätzen aus Smart Metern mit enormer Datentiefe verglichen und erweitert werden.

Ein ebenfalls probabilistischer Ansatz wird bei der Netzsicherheitsbewertung beschritten: Hierbei werden Maßzahlen zur Risikobeurteilung eines gegebenen Netzzustandes bestimmt, deren Aussagekraft über das bisher übliche $n-1$-Kriterium weit hinausgeht.

Der Trend zur Urbanisierung bedeutet für den Ausbau von Leitungsnetzen, dass bei Neubauten in Ballungsgebieten ausschließlich Kabel eingesetzt werden, welche im Hinblick auf andere Infrastruktur-Einrichtungen einige kritische Eigenschaften aufweisen: Der entsprechende Beitrag analysiert die Problematik der Län- genänderung bei in Bauwerken verlegten Hochspannungskabeln und skizziert Lösungsansätze zur deren Beherrschung.

Elektrische Energiesysteme sind aber nicht nur für den stationären Normalbetrieb auszulegen, sondern müssen auch bei möglichen Netzfehlern Resilienz aufweisen. Lange Zeit waren die Kurzschlussberechnung und das Schutzgeschehen von klaren und eindeutigen Stromflüssen gekennzeichnet. Durch den zunehmenden Einsatz nichtrotierender Energiewandler in Form von Wechselrichtern ist allerdings keine eindeutige Fehlerstromvorhersage mehr möglich, weshalb neue Schutzformen und resilienzstärkende Maßnahmen erforderlich werden.

Das menschliche Leben als höchstes Rechtsgut ist besonders zu schützen, und daher genießen personenschutz-orientierte Fragestellungen des elektrischen Fehlergeschehens besondere Beachtung. In der "kritischen Betrachtung des Löschverhaltens in kompensierten 20-kV-Netzen" wird die in vielen Anwendungen als Designwert herangezogene "Löschgrenze" im historischen Kontext noch einmal kritisch durchleuchtet und ein neuartiger, auf physikalischen Tatsachen basierender funktioneller Zusammenhang erarbeitet.

Alternde Infrastruktur bedeutet bei elektrischen Kabelleitungen vor allem die Abnahme der Isolationsfestigkeit und erfordert damit neue Lösungen beim Umgang mit ein- und mehrpoligen Isolationsfehlern. Als isolationsschonende und betriebsfreundliche Verfahren der Sternpunktbehandlung gewinnt die Klasse der kurzzeitig stromstarken Ortungsverfahren an Attraktivität: Die sogenannte KNOPE wird im sechsten Beitrag sowohl hinsichtlich der Auslegung als auch der Implementierung beschrieben, weiters werden erste Praxiserfahrungen wiedergegeben.

Ebenfalls der Erdschlussproblematik gewidmet ist die Untersuchung der Stromaufteilung im Nullsystem entlang des Fehlerpfades, wobei wichtige und hinsichtlich der Beschreibung globaler Erdungssysteme zukunftweisende Ergebnisse aus einer Serie von Erdschlussversuchen in einem Mittelspannungsnetz gewonnen werden.

Große Übertragungsnetze stehen in Zeiten abnehmender Schwungmassen vor Stabilitätsproblemen. Drei Beiträge befassen sich mit dieser Herausforderung: Der erste untersucht Netzpendelungen und ihre verschiedenen Oszillationsmoden mit dem Ziel der berechnungstechnischen Vereinfachung, der zweite geht den Aufgaben und Möglichkeiten von Low-Voltage-Ride-ThroughAssistenzsystemen für dezentrale Erzeugungseinheiten nach. Der dritte Aufsatz vergleicht verschiedene netzstabilisierende Technologien in einem HGÜ-gespeisten Inselnetz hinsichtlich ihrer Effektivität bei der Blindleistungsbereitstellung und ihrem Verhalten im Fehlerfall.

Fickert, Lothar, Technische Universität Graz, Institut für Elektrische Anlagen, Inffeldgasse 18/1, 8010 Graz, Österreich (E-Mail: lothar.fickert@tugraz.at) 
Durch den vermehrten Netzausbau und die daraus resultierenden räumlichen Annäherungen von Hochspannungsfreileitungssystemen und -kabelsystemen in so genannten Energiekorridoren werden Beeinflussungsanalysen und die Bestimmung von induktiven Beeinflussungen zunehmend wichtiger: Der erste Beitrag auf diesem Themengebiet beschreibt einen über die vereinfachten Berech- nungsmodelle sowie klassische Beeinflussungsberechnungsmethoden hinausgehenden Ansatz auf Basis des Knotenpotentialverfahrens, der zweite behandelt das Thema der ohmschen und induktiven Beeinflussung sowie deren Auswirkungen bei HöchstspannungsKabelsystemen, um daraus effizientere Berechnungsmethoden abzuleiten. 